\title{
Cutaneous manifestations of Churg-Strauss syndrome: key to diagnosis*
}

\author{
Camila Carneiro Marques ${ }^{1}$ \\ Gabriela Momente Miquelin ${ }^{1}$
}

\author{
Elizabeth Leocadia Fernandes ${ }^{1}$ \\ Mariana Morais Tavares Colferai ${ }^{1}$
}

DOI: http:/ / dx.doi.org/10.1590/abd1806-4841.20175522

\begin{abstract}
Churg-Strauss syndrome is a rare systemic vasculitis characterized by asthma and other allergy symptoms as well as eosinophilia and necrotizing vasculitis involving small and medium-sized vessels. Its prevalence in the general population ranges from 1-3 cases per million a year, varying according to the population studied. The authors describe a case of a female patient affected by the disease with important systemic manifestations and not very florid skin lesions.
\end{abstract}

Keywords: Skin manifestations; Churg-Strauss Syndrome; Vasculitis

\section{INTRODUCTION}

Churg-Strauss syndrome (CSS), or eosinophilic granulomatosis with polyangiitis, is a rare systemic vasculitis characterized by asthma and other allergic symptoms, besides eosinophilia and necrotizing vasculitis of small and medium vessels. ${ }^{1}$ The prevalence in the general population is of 1-3 cases/million, and varies according to the population studied. The condition affects both sexes with the same frequency, in the third and fifth decades of life. ${ }^{2}$

\section{CASE REPORT}

A 53-year-old female patient presented at the dermatology service with history of fever, slightly pruritic lesions on the skin, and arthralgia for two months, besides hypoesthesia and reduced muscle strength of the left leg and foot, and areas of hyperesthesia on the left foot for one month. With this clinical picture, the patient went multiple times to the emergency department and was treated with corticoids, nonsteroidal anti-inflammatory drugs, antibiotics and analgesics. She was also assessed by an infectious disease specialist and a cardiologist. She finally went to a rheumatologist, who referred her to the dermatology service.

The patient had asthma for four years, was followed by a respiratory physician and was on Montelukast, corticoid inhaler and long acting beta2-agonist. There was no history of rhinitis.

On dermatologic examination, there were erythematous, purpuric papules, with overlying erosions or crusts on the dorsum of the hand, on the index finger, and on the left elbow and foot (Figures 1 to 3). There was also hypoesthesia and areas of loss of tactile, pain and thermal sensation on the outer edge of the left leg and all over the left foot.

\footnotetext{
Study submitted on 21.12.2015

Approved by the Advisory Board and accepted for publication on 01.08.2016

* Study conducted at the Dermatology Outpatient Clinic, Universidade de Mogi das Cruzes - Mogi das Cruzes (SP), Brazil.

Financial support: None

Conflict of interest: None
}

1 Dermatology Outpatient Clinic, Universidade de Mogi das Cruzes - Mogi das Cruzes (SP), Brazil. 
Laboratory tests showed leukocytosis $\left(16840 / \mathrm{mm}^{3}\right)$, with $25,70 \%$ eosinophils $\left(4320 / \mathrm{mm}^{3}\right)$, and creatinine $0.7 \mathrm{mg} / \mathrm{dL}$. Urinalysis showed mild hematuria with no proteinuria.

Further laboratory tests showed positive p-ANCA. Electroneuromyography revealed post-ganglion axonal neurogenic damage, partially affecting the left inferior lumbosacral trunk. The neuropathy was in the acute phase, with active signs of recent denervation and reinnervation. As for the other organs and systems, the echocardiogram showed reduced systolic function (ejection fraction: $48 \%$ ) and mild diastolic dysfunction. Despite the history of asthma, there were no pulmonary infiltrates on chest tomography.

The picture of asthma, fever, multiple mononeuropathy and eosinophilia, associated to sparse papular-purpuric lesions lead to the suspicion of CSS. A biopsy was taken from the patient's left foot, and the histology shown in Figure 4, showed multiple changes of small vessels with fibrin deposition and endothelial swelling, besides leukocytoclasis and malformed granulomas with multiple

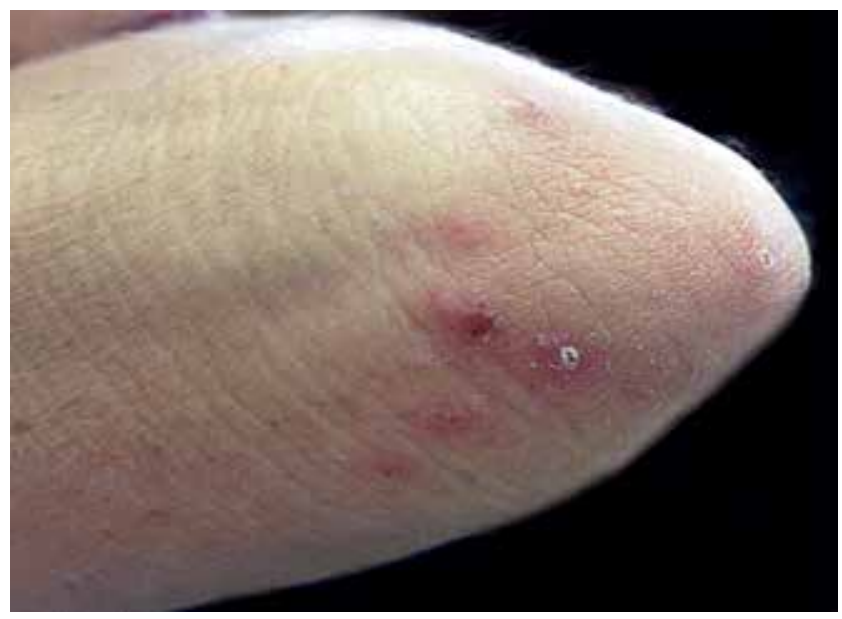

FiguRE 1: Erythematous, purpuric papules with overlying erosions and crusts on the elbow

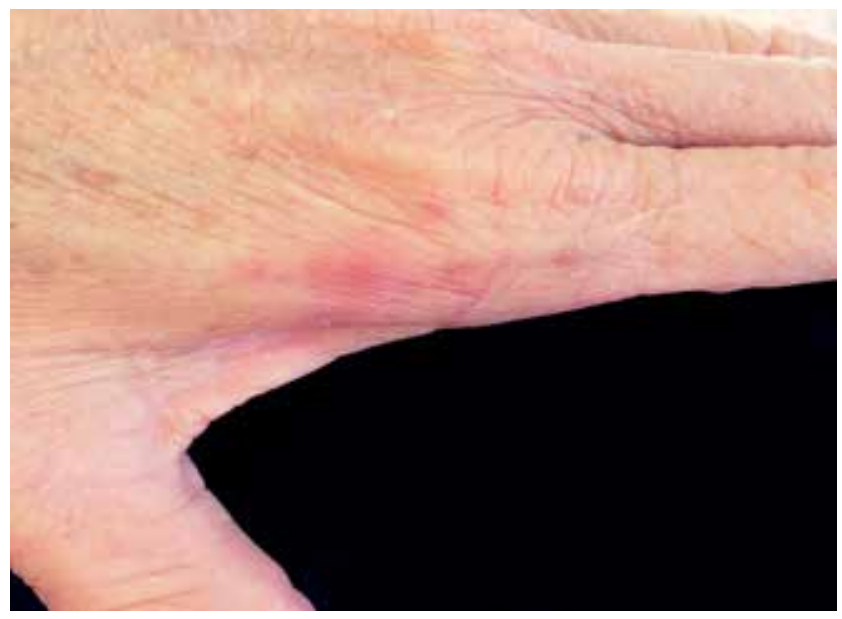

FigURE 2: Erythematous, purpuric papules on the dorsum of the left hand conglomerates of eosinophils. Such features supported the hypothesis of CSS.

Treatment was started with prednisolone $(60 \mathrm{mg} /$ day) and cyclophosphamide pulse therapy. There was obvious clinical improvement with cessation of fever and arthralgia and improvement of the muscle strength of the left foot; the only remaining change was the impaired sensation on the left malleolus. The leukocytosis $\left(8320 / \mathrm{mm}^{3}\right)$ and the eosinophilia $\left(83 / \mathrm{mm}^{3}\right)$ disappeared.

\section{DISCUSSION}

CSS is characterized by the onset of vasculitis, that manifests as multiple mononeuropathy, purpura, general symptoms and eosinophilia in a patient with previous asthma. However, some patients can develop asthma or eosinophilia and vasculitis simultaneously. ${ }^{1}$

The initial and most common manifestations of CSS include the involvement of the respiratory tract with asthma and allergic rhinosinusitis, with possible nasal polyps, pulmonary infiltrates

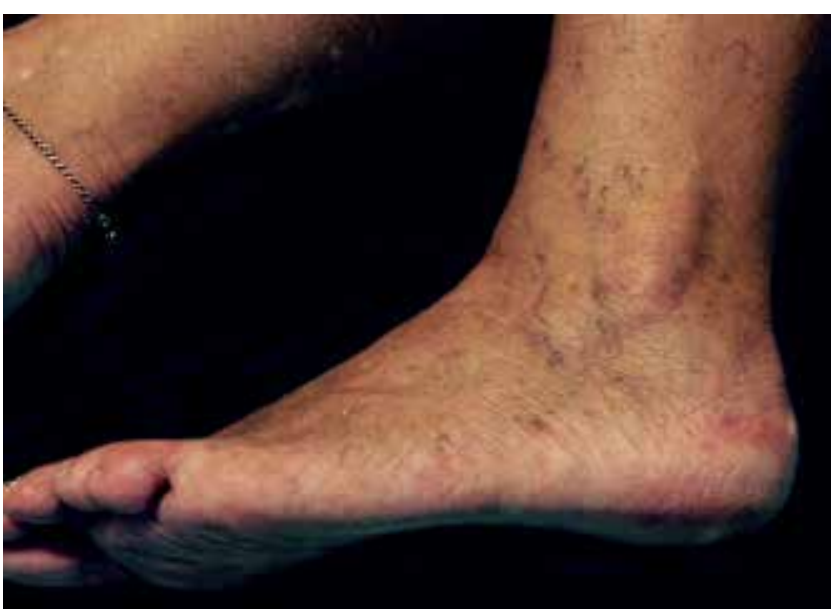

Figure 3: Erythematous, purpuric papules on the lateral aspect of the left foot

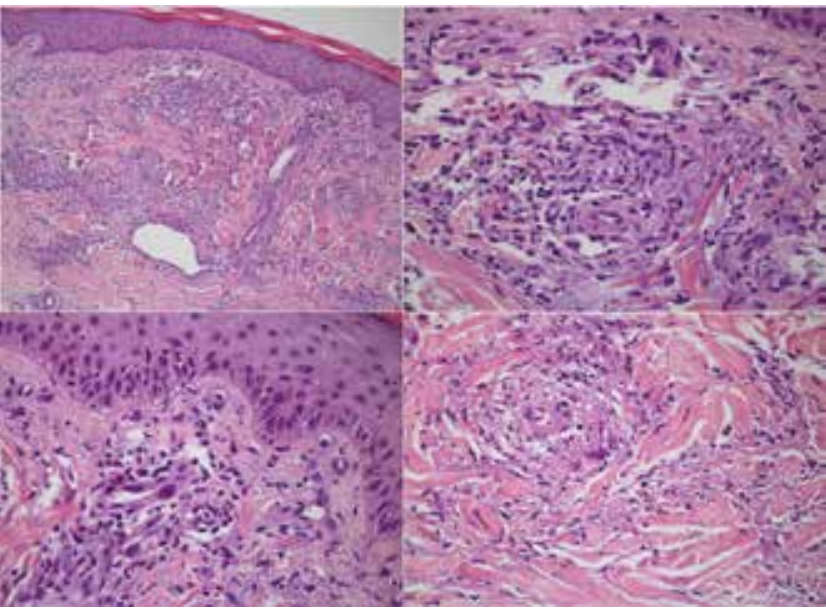

Figure 4: Multiple changes of the small vessels with foci of fibrin deposition. Leukocytoclasis. Malformed granuloma with interspersed eosinophils (Hematoxylin \& eosin, X20 and X400) 
and pleural effusion. Peripheral neuropathy, manifested by multiple mononeuropathy, occurs in a third of the cases. Renal involvement affects about $30 \%$ of patients and can cause urinary abnormalities, including rapidly progressive renal failure. There is gastrointestinal involvement in a third of patients, that can present with abdominal pain, nausea, vomits, diarrhea, intestinal bleeding and acute abdomen. ${ }^{3}$ Cardiovascular system is affected in about $10 \%$ of cases and this involvement is the primary cause of death in patients with CSS. Among the most important manifestations are coronary arteritis and myocarditis. ${ }^{4}$ Although rare, central nervous system involvement is the major cause of mortality among patients. ${ }^{3}$

About $50 \%$ of patients will present with skin changes. These include: 1) hemorrhagic lesions, such as palpable purpuras, petechiae, ecchymosis and hemorrhagic bullae, and 2) dermal or subcutaneous papules and nodules, frequently located on the scalp or distributed bilaterally over the extensor surfaces of the extremities. Other changes that can also be observed less frequently are urticaria, erythematous macules and livedo reticularis. ${ }^{5}$

CSS has three evolutionary phases. In the first phase, that can last years or decades, only respiratory manifestations such as asthma, rhinitis and nasal polyps occur. In the second phase, there is peripheral and tissue eosinophilia, affecting primarily the lungs, intestines and myocardium. In the third phase, there is systemic vasculitis affecting nerves, kidneys and skin. ${ }^{3}$

CSS diagnostic criteria defined by the American College of Rheumatology (ACR) are described in Chart 1.

Despite the mild cutaneous manifestations of the patient, there were four undisputable diagnostic criteria present: history of asthma, eosinophilia, neuropathy and vascular eosinophilic infiltrate on the histology. Besides, p-ANCA was positive, making it possible to diagnose the third phase of CSS.

CSS prognosis is variable and depends on the initial extension of the disease and the organs affected. Five factors were described that are associated to a higher mortality among patients;
ChART 1: Diagnostic criteria for Churg-Strauss syndrome (American College of Rheumatology)

Asthma

Eosinophilia (> 10\%)

Paranasal sinusitis

Pulmonary infiltrate

Biopsy showing vascular eosinophilic infiltrate

Multiple mononeuropathy or polyneuropathy

Three or more criteria: sensitivity of $85 \%$ and specificity of $99.7 \%$

which are: creatinine higher than $1.58 \mathrm{mg} / \mathrm{dL}$, proteinuria higher than $1 \mathrm{~g} /$ day, gastrointestinal involvement, central nervous system involvement and cardiomyopathy. ${ }^{6}$ These criteria help identify the patients that need a more aggressive immunosuppressive therapy, due to the higher chance of mortality and higher frequency of relapse. Conventional treatment includes the use of corticosteroids. For the patients that are unresponsive to corticosteroid therapy or to those that have poor prognosis features, immunosuppressive therapy with cytotoxic agents is indicated. Cyclophosphamide is the most effective cytotoxic to induce CSS remission. ${ }^{7}$ We opted to use cyclophosphamide because the patient failed corticosteroids, that were used for months for her asthma. Besides, she already had mild cardiac systolic dysfunction.

Cutaneous manifestations of CSS are one type of clinical presentation for this condition. Recognition of these lesions by the dermatologist was essential for the clinical suspicion and confirmation of diagnosis, that allowed adequate treatment, reducing morbidity and contributing for the prevention of irreversible lesions in vital organs.]

\section{REFERENCES}

1. Mouthon L, Dunogue B, Guillevin L. Diagnosis and classification of eosinophilic granulomatosis with polyangiitis (formerly named Churg-Strauss syndrome). J Autoimmun. 2014;48-49:99-103.

2. Watts RA, Lane SE, Bentham G, Scott DG. Epidemiology of systemic vasculitis: a ten-year study in the United Kingdom. Arthritis Rheum. 2000;43:414-9.

3. Keogh KA, Specks U. Churg-Strauss Syndrome: update on clinical, laboratory and therapeutic aspects. Sarcoidosis Vasc Diffuse Lung Dis. 2006;23:3-12.

4. Lestre S, Serrão V, João A, Pinheiro S, Lobo L. Síndrome de Churg-Strauss vasculite cutânea como forma de apresentação clínica. Acta Reumatol Port. 2009;34:281-7

5. Chen KR, Carlson JA. Clinical approach to cutaneous vasculitis. Am J Clin Dermatol. 2008;9:71-92.

6. $\quad$ Kim MY, Sohn KH, Song WJ, Park HW, Cho SH, Min KU, et al. Clinical features and prognostic factors of Churg-Strauss syndrome. Korean J Intern Med. 2014;29:85-95

7. Taniguchi M, Tsurikisawa N, Higashi N, Saito H, Mita H, Mori A, et al. Treatment for Churg-Strauss Syndrome: Induction of Remission and Efficacy of Intravenous Immunoglobulin Therapy. Allergol Int. 2007;56:97-103.

\author{
MAILING ADDRESS: \\ Camila Carneiro Marques \\ Rua Dom Antônio Cândido Alvarenga, 170 \\ Centro \\ 08780-070 Mogi das Cruzes, SP \\ Brazil \\ E-mail:cam_tn1@hotmail.com
}

How to cite this article: Marques CC, Fernandes EL, Miquelin GM, Colferai MMT. Cutaneous manifestations of Churg-Strauss syndrome: key to diagnosis. An Bras Dermatol. 2017;92(5 Suppl 1): 56-8. 